\title{
Body weight status of children aged 7 to 9 in France since 2000: evolution and associated factors
}

\section{Abstract}

Introduction: Overweight and obesity are major risk factors for non-communicable diseases and their prevalence is spreading in highincome countries. However, in France, after an increase among children until 2000, a stabilization of overweight was observed in two national studies conducted by The French Public Health Agency in 2000 and 2007. The study was renewed in 2016 as part of the Children Obesity Surveillance Initiative (COSI) of the WHO regional office for Europe. This work describes the evolution of the prevalence of overweight among children during the period and the relation between overweight and associated factors in 2016.

Materials and methods: The three studies, conducted according to a similar protocol, concerned pupils aged 7 to 9 , in primary schools randomly selected throughout the metropolitan area. Weight and height of the children were measured by the nursing staff of the ministry of Education. A self-questionnaire completed by the parents collected sociodemographic characteristics. Prevalence of overweight and obesity was calculated according to the thresholds of the International Obesity Task Force. Analysis was performed using weighting to take into account the complex sampling scheme, and using calibration to provide nationally representative results. Weighting was calculated separately between Education priority area and non-priority area, which depends on the socioeconomic level of the neighborhood.

Results: While the stabilization of overweight (including obesity) observed since 2000 was confirmed among girls ( $21.0 \%$ in 2007 versus $18.7 \%$ in 2016), a significant decrease was observed among boys (from $18.0 \%$ in 2007 to $14.4 \%$ in 2016 , p = 0.046). In 2016, the gap between girls and boys was significant $(\mathrm{p}<0.01)$ for the first time compared to previous results obtained in 2000 and 2007 . The prevalence of obesity was also significantly higher in 2016 among girls than boys $(5.5 \%$ versus $3.2 \%, p=0.01)$. This excess risk of overweight or obesity in 2016 persisted between genders even after adjusting for covariates. Among children from Education priority area, $21.0 \%$ were overweight or obese versus $15.7 \%$ in children living in non-priority area $(p=0.001)$.

Discussion: These results confirm the stabilization of overweight among girls and show, for the first time, a decrease in boys. They also highlight gender and social inequalities in children in France. These trends will need to be confirmed by other studies, and analyzed in more detail to understand the reasons for these differentiated evolutions between genders.

\section{Conflict of Interest}

There is no conflict of interest 